\title{
Desigualdad y Política Fiscal
}

\author{
En estos tiempos, caracterizados por un incremento generalizado \\ de la desigualdad, este artículo hace una revisión de la literatura \\ existente sobre el papel que puede jugar la política fiscal para reducirla. \\ Comenzaremos con los resultados generales más relevantes, seguiremos \\ con la capacidad redistributiva de los diferentes instrumentos fiscales y \\ terminaremos con los efectos redistributivos de la consolidación fiscal.
}

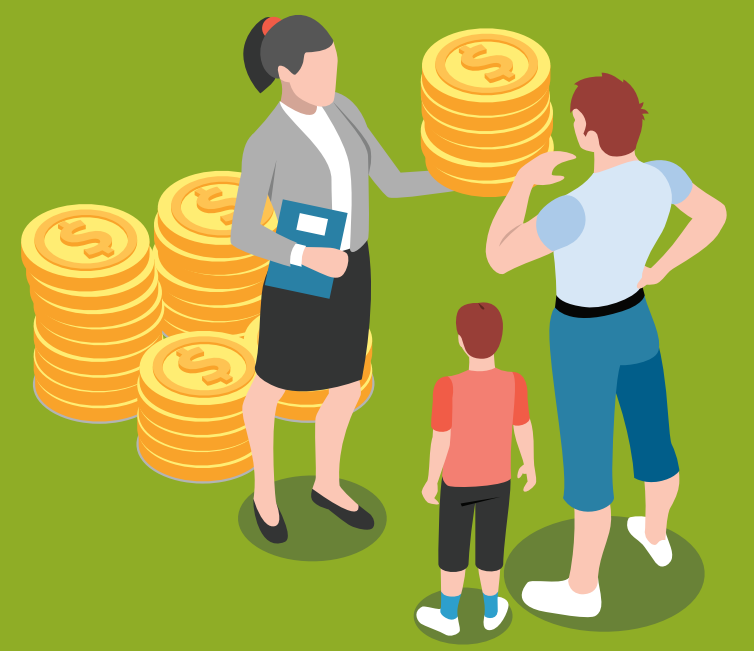

\section{RESULTADOS GENERALES}

os resultados generales son múltiples, en ocasiones contradictorios, lo que no deja de ser una medida de lo alejados que aún estamos de obtener resultados realmente convincentes.

Algunos estudios encajan bien en una visión positiva de la política fiscal. Por ejemplo, Onrubia y Rodado (2014) afirman que la evolución de la desigualdad de las rentas de mercado muestra que el funcionamiento de la economía, sin mecanismos redistributivos, genera un aumento en el tiempo de la desigualdad de la renta. De este modo, la ausencia de un sistema fiscal redistributivo, corrector de esta desigualdad antes de la intervención estatal, agravaría esta situación.

No obstante, la capacidad redistributiva de los sistemas fiscales varía con el tiempo. Por ejemplo, eran más efectivos en reducir la desigualdad a mediados de los 2000 que a mediados de los 90 (Wang et al., 2014). Un resultado confirmado por los resultados de Immervoll y Richardson (2011), quienes afirman que, en el contexto de la creciente desigualdad de los ingresos de mercado, los sistemas de transferencias e impuestos se han convertido en más redistributivos desde los años 80 , aunque esto no detuvo el crecimiento de la desigualdad: la desigualdad de los ingresos de mercado creció el doble que la redistribución.
Sin embargo, tanto Woo et al (2013) como Bastagli et al (2012) afirman que las reformas producidas desde mediados de los 90 han menguado la generosidad de las transferencias sociales y la progresividad de los sistemas impositivos sobre el ingreso en las economías avanzadas, haciendo la política fiscal menos redistributiva. Las causas de este menor poder redistributivo de la política fiscal en las economías desarrollados (FMI, 2014) son las reformas fiscales que han tenido lugar en muchos países desde mediados de los noventa, que han reducido la generosidad de las ayudas sociales y al desempleo, así como los tipos del impuesto sobre el ingreso, especial-

Resumen de la literatura que estudia la relación entre Desigualdad y Política Fiscal

\begin{tabular}{|c|c|c|}
\hline Investigación & Resultados & Autores \\
\hline \multirow{3}{*}{$\begin{array}{l}\text { Resultados } \\
\text { generales }\end{array}$} & Visión positiva & $\begin{array}{l}\text { Onrubia y Rodado (2014); Wang et al } \\
\text { (2014); Immervoll y Richardson (2011) }\end{array}$ \\
\hline & Visión negativa & $\begin{array}{l}\text { Woo et al (2013); Bastagli et al (2012); } \\
\text { FMI (2014); Föster et al (2014) }\end{array}$ \\
\hline & Indiferencia & $\begin{array}{l}\text { Casalone y Sonedda (2013); Martínez- } \\
\text { Vázquez et al (2012) }\end{array}$ \\
\hline \multirow{4}{*}{$\begin{array}{l}\text { Efectos } \\
\text { redistribuidos } \\
\text { individuales }\end{array}$} & $\begin{array}{l}\text { Las transferencias son } \\
\text { más redistribuidas }\end{array}$ & Rodrigues y Andrade (2014) \\
\hline & $\begin{array}{l}\text { Las pensiones y las } \\
\text { prestaciones por desempleo }\end{array}$ & $\begin{array}{l}\text { Cantó (2013); Niehues (2010); Wang et } \\
\text { al (2014); OCDE (2012) }\end{array}$ \\
\hline & Educación y sanidad & $\begin{array}{l}\text { Ospina (2010); Spadaro (2011); } \\
\text { Bastagli et al (2012) }\end{array}$ \\
\hline & $\begin{array}{l}\text { El papel redistributivo de los } \\
\text { impuestos: El impuesto sobre } \\
\text { el ingreso personal y el gasto } \\
\text { social }\end{array}$ & $\begin{array}{l}\text { Martínez-Vázquez et al (2012); } \\
\text { Verbist y Figari (2014) }\end{array}$ \\
\hline \multirow{2}{*}{$\begin{array}{l}\text { Las políticas de } \\
\text { consolidación } \\
\text { fiscal }\end{array}$} & Aumento de la desigualdad & $\begin{array}{l}\text { Matsaganis y Leventi (2014); } \\
\text { Rodrigues y Andrade (2014); Bastagli } \\
\text { et al (2013); Ball et al (2013) }\end{array}$ \\
\hline & Efecto ambiguo & $\begin{array}{l}\text { Bargain (2017); FMI (2014); Woo et al } \\
\text { (2013); Agnello y Sousa (2012) }\end{array}$ \\
\hline
\end{tabular}

Fuente: Elaboración propia. 
mente a los ingresos más altos. Föster et al (2014) también confirman una considerable reducción en los tipos impositivos marginales de los más ricos durante las pasadas décadas, así como la existencia de una fuerte correlación negativa entre dichos tipos impositivos y sus proporciones de ingresos, en los países de la OCDE.

No obstante, los resultados de otros autores defienden un papel redistributivo casi nulo de la política fiscal. Por ejemplo, la principal conclusión de Casalone y Sonedda (2013) es que las reformas fiscales que tuvieron lugar en Italia durante el año 2004 no tuvieron repercusiones redistributivas alguna. En la misma línea, para Martínez-Vázquez et al (2012) el impacto de la política fiscal, en conjunto, ha sido muy limitado.

\section{EFECTOS REDISTRIBUTIVOS DE LOS DIFERENTES INSTRUMENTOS FISCALES}

La mayoría de los estudios concluyen que el gasto público es más redistributivo que los impuestos. Por ejemplo, para Rodrigues y Andrade (2014), todos los instrumentos de política social y fiscal analizados contribuyen significativamente a reducir la desigualdad y la pobreza, pero son las transferencias las más eficientes en términos de costes. No obstante, no todo el gasto resulta igualmente redistributivo, destacando claramente el sistema de pensiones y las prestaciones por desempleo. Por ejemplo, en el estudio que Cantó (2013) hace sobre la capacidad redistributiva del sistema fiscal español, concluye que más de la mitad del efecto redistributivo de todo el sistema se debe a las pensiones, aunque se ha reducido durante los últimos años del periodo de estudio (2005-2011). Sin embargo, este menor efecto igualador de las pensiones se ha visto compensado por una mayor capacidad redistributiva del impuesto sobre la renta. La fuerte capacidad redistributiva del sistema de pensiones (además de las prestaciones por el desempleo) es también corroborada por las conclusiones de Niehues (2010), quien defiende que un mayor gasto social está fuertemente relacionado con menor desigualdad en los ingresos disponibles. No obstante, las ayudas dirigidas a los grupos de más bajos ingresos no están relacionadas con menor desigualdad, lo que sugiere la existencia de posibles efectos desincentivos que contrarrestan los efectos igualitarios de primer orden. Los resultados de Wang et al. (2014) también defienden al sistema de pensiones como el principal instrumento redistributivo. Para ellos dicho sistema constituyeron el $60 \%$ del incremento en la redistribución producido durante las últimas décadas, mientras que las ayudas sociales contabilizaron el 20\%. Según OCDE (2012), las transferencias monetarias (tales como las pensiones, las ayudas al desempleo y a la infancia), contabilizan más de países. Aunque para ellos el impacto redistributivo total de la política fiscal está también influenciado por los impuestos indirectos (que tienden a incrementar la desigualdad) y por las transferencias en especie (tales como la educación y la sanidad).

También hay autores que resaltan el papel redistributivo de los impuestos. En este sentido, para Martínez-Vázquez et

\section{"Hay autores que resaltan el papel redistributivo de los impuestos. En este sentido, para Martínez-Vázquez et al (2012), el impuesto sobre el ingreso personal (este es el impuesto más progresivo, según Verbist y Figari, 2014) y el gasto social, en general, reducen la desigualdad; mientras que las cuotas de la seguridad social, el IVA y otros impuestos indirectos la aumentan"}

tres cuartas partes del impacto redistributivo total en la OCDE y los impuestos contabilizan el resto.

Otros gastos calificados también como de especialmente redistributivos son los de educación y sanidad. Por ejemplo, una vez que el problema de endogeneidad de las variables es controlado, según Ospina (2010), el gasto en educación y sanidad tiene un efecto beneficioso sobre la desigualdad del ingreso, mientras que el gasto en seguridad social no tiene efecto alguno. Tanto el gasto sanitario como el educativo reducen la desigualdad en los países en desarrollo, aunque es más efectivo el gasto en educación. Sin embargo, estudios análogos han sobrestimado los efectos de ambos tipos de gasto, debido al uso de métodos econométricos inapropiados. El efecto progresivo del gasto sanitario sobre la desigualdad es confirmado por el estudio que Spadaro et al (2011) hacen sobre dicho gasto en España, mostrando una buena capacidad para sacar a las familias de la pobreza. Bastagli et al (2012) también califican al gasto en educación y sanidad como de altamente redistributivos. Para ellos, la mayoría del impacto redistributivo de la política fiscal es alcanzado por el lado del gasto del presupuesto, especialmente las transferencias sin evaluación de medios (pensiones y transferencias universales a la infancia), aunque los impuestos sobre el ingreso son también importantes en muchos al (2012), el impuesto sobre el ingreso personal (este es el impuesto más progresivo, según Verbist y Figari, 2014) y el gasto social, en general, reducen la desigualdad; mientras que las cuotas de la seguridad social, el IVA y otros impuestos indirectos la aumentan. Algunos impuestos tienen un mayor efecto adverso sobre la actividad económica que otros. En este sentido, los impuestos sobre el ingreso personal y corporativo suelen ser los más distorsionadores (OCDE, 2012). En general, los impuestos directos (por ejemplo, el impuesto sobre el ingreso personal $y$, en menor medida, el impuesto sobre el ingreso corporativo) y el gasto social mejoran la distribución del ingreso; mientras que los impuestos indirectos (incluyendo, los impuestos sobre el consumo) tienden a incrementar la desigualdad (Woo et al, 2013).

\section{LOS EFECTOS REDISTRIBUTIVOS DE LAS POLÍTICAS DE CONSOLIDACIÓN FISCAL}

De la revisión de literatura que Matsaganis y Leventi (2014) llevan a cabo deducen tres ideas claves: (1) los periodos de consolidación fiscal tienden a estar asociados con aumentos en la desigualdad, (2) los efectos estáticos de las políticas de austeridad pueden ser compensados por los efectos dinámicos, (3) el diseño de los paquetes de consolidación fiscal puede neutralizar, parcial o completamente, los 
efectos distributivos adversos. Aunque el impacto de las políticas sobre la desigualdad puede ser descrito como moderado (o incluso pro-igualdad), esto no equivale a decir que los programas de ajuste fiscal hayan tenido éxito en términos distributivos. Las estimaciones de estos autores sugieren que en la mayoría de los países examinados la pobreza aumentó, y las políticas implementadas jugaron un papel importante en dicho incremento. En esta línea de ambigüedad, se definen también otros autores, como Bargain et al (2017), quienes afirman que las medidas adoptadas por varios países desarrollados para combatir los desequilibrios fiscales no aumentaron la desigualdad ni la pobreza, incluso en algunos países las redujeron. También podríamos incluir en esta línea a Jenkins et al (2011), quienes afirman que durante los años siguientes al estallido de la crisis, la desigualdad no se ha visto significativamente afectada, pero a medio y largo plazo, probablemente habrá grandes cambios en los niveles de desigualdad, como consecuencia de las medidas de consolidación fiscal que están siendo implementadas.

No obstante, para Rodrigues y Andrade (2014) el impacto de la crisis económica y las políticas de austeridad implementadas desde 2010 en Portugal, han invertido las tendencias anteriores y han afectado negativamente la eficacia y la eficiencia de todos los instrumentos fiscales. En este sentido, según FMI (2014), en las economías avanzadas, la composición y el ritmo de la consolidación fiscal condicionan su impacto sobre la desigualdad. Sus resultados demuestran que los ajustes fiscales basados en los recortes del gasto empeoran la desigualdad más que los ajustes basados en los ingresos. Su análisis sugiere que tuvieron un carácter progresivo los recortes en los salarios del sector público, los recortes en las transferencias no dirigidas, los incrementos en el impuesto sobre el ingreso y las contribuciones sociales; mientras que tuvieron un efecto regresivo los recortes en las transferencias dirigidas, las reducciones generales y proporcionales en las pensiones, y los incrementos en los tipos del IVA.

El carácter más perjudicial de los recortes del gasto también es defendido por Woo et al (2013). La causa de esto parece estar en que los ganadores de ingresos más bajos suelen estar más afectados por los recortes en el gasto público, ya que una parte importante de sus ingresos provienen del mismo y ellos son más vulnerables a las pérdidas del empleo. La misma idea es defendida por Agnello y Sousa (2012), incluso afirman que los ajustes basados en incrementos de los impuestos suelen tener un efecto igualador. No obstante, también afirman que el momento de aplicación de los ajustes resulta decisivo. Cuando tiene lugar durante una crisis bancaria, el impacto sobre la desigualdad suele ser insignificante, pero si tiene lugar cuando dicha crisis ya ha sido resuelta, la consolidación fiscal aumenta considerablemente la desigualdad del ingreso. En esta misma línea se sitúan Ball et al (2013), cuyo resultado principal es que los ajustes fiscales normalmente han tenido efectos redistributivos significativos, aumentando la desigualdad, disminuyendo la proporción de salarios e incrementando el desempleo a largo plazo. Sin embargo, para Bastagli et al (2012) dicho incremento se produce a corto plazo. De hecho, para estos autores existen dos formas por las que la consolidación fiscal afecta a la distribución del ingreso. Una es la reducción de la producción y el aumento del desempleo, lo que se traduce normalmente en una disminución de la proporción de salarios; la segunda la constituyen los cambios en los niveles y en la composición de los impuestos y del gasto público.

\section{BIBLIOGRAFÍA}

Agnello, L. y Sousa, R. (2012). How does Fiscal Consolidation Impact on Income Inequality? Banque de France, Working Paper n 382

Ball, L.; Furceri, D.; Leigh, D. y Loungani, P. (2013). The Distributional Effects of Fiscal Consolidation. International Mnetary Fund, Working Paper 13/151.

Bargain, O; Callan, T.; Doorley, K. y Keane, C. (2017). Changes in Income Distributions and the Role of Tax-Benefit Policy During the Great Recesion: An International Perspective. Fiscal Studies, 38(4), 559-585.

Bastagli, F; Cpady, D. y Gupta, S. (2012). Income Inequality and Fiscal Policy. International Monetary Fund, SDN/12/08.

Cantó, O. (2013). La capacidad redistributiva del sistema español de prestaciones e impuestos. Papeles de Economía Española, 135, $140-152$.

Casalone, G. y Sonedda, D. (2013). Evaluating the distributional effects of fiscal policies using quantile regressions. Review of Income and Wealth, 59(2), 305-325.

FMI (2014). Fiscal Policy and Income Inequality. IMF Policy Paper.

Förster, M.; Llena-Nozal, A. y Nafilyan, V. (2014). Trends in Top Incomes and their Taxation in OECD Countries. OECD Social Employment and Migration Working Papers, 159, $1-93$.

Immervoll, H. y Richardson, L. (2011). Redistribution Policy and Inequality Reduction in OECD Countries: What Has Changed in Two Decades? Institute for the Study of Labor (IZA), Discussion Paper № 6030.

Jenkins, S.; Brandolini, A.; Micklewright, J. y Nolan, B. (septiembre, 2011). The Great Recession and the Distribution of Household Income. Trabajo presentado en Incomes Across the Great Recession, XIII European Conference of the Fondazione Rodolfo Debenedetti, Palermo.

Martínez-Vázquez, J.; Vulovic, V. y Moreno-Dodson, B. (2012). The Impact of Tax and Expenditure Policies on Income Dsitribution: Evidence from a large panel of countries. Hacienda Pública Española, 200(4), 95-130.

Matsaganis, M. y Leventi, C. (2014). Distributive Effects of the Crisis and Austerity in seven EU Countries. Working Papers. IMPROVE Poverty Social Policy and Innovation, $14 / 04$.

Niehues, J. (2010). Social Spending Generosity and Income Inequality: ADynamic Panel Approach, Beiträge zur Jahrestagung des Vereins für Socialpolitik. Ökonomie der Familie - Session: Distribution and Redistribution, No. F14-V3

OECD (2012). Income inequality and growth: The role of taxes and transfers. OECD Economics Department Policy Notes, No. 9.

Onrubia, J. y Rodado, M. (2014). Presión fiscal en España. Análisis de la presión fiscal por niveles de renta en España, 2009-2011. Informes de Investigación de OXFAM INTERMÓN, Mayo 2014.

Ospina, M. (2010). The Effect of Social Spending on Income Inequality: An Analysis for Latin American Countries. Centro de investigaciones Económicas y Financieras (CIEF) Universidad EAFIT, n 10-03.

Rodrigues, C. y Andrade, I. (2014). Robin Hood versus Piggy Bank: Income Redistribution in Portugal 2006-2010. PANOECONOMICUS, 61(5), 617-630.

Spadaro, A.; Moral, I.; Adiego, M.; Blanco, A. y Mangiavacchi, L. (2011). Evaluating the Redistributive Impact of Public Health Expenditure using an Insurance Value Approach. The European Journal of Health Economics, 14(5), 775-787.

Verbist, G. y Figari, F. (2014). The redistributive effect and progressivity of taxes revisited: An international comparison across the European Union, EUROMOD Working Paper, No. EM6/14

Wang, C; Caminada, K. y Goudswaard, K. (2014). Income redistribution in 20 countries over time. International Journal of Social Welfare, 23, $262-275$.

Woo, J.; Bova, E.; Kinda, T. y Zhang Y.S. (2013). Distributional Consequences of Fiscal Consolidation and the Role of Fiscal Policy: What Do the Data Say? International Monetary Fund, Working Paper 13/195. 